\title{
Inhibitory effect of soluble RAGE in disturbed flow-induced atherogenesis
}

\author{
CHANG HOON HA ${ }^{1 *}$, SUNGHYEN KIM ${ }^{1 *}$, JIHWA CHUNG $^{1}$, SHUNG HYEN AN $^{1}$, \\ SUNGHA PARK ${ }^{3}$, DONGHOON $\mathrm{CHOI}^{3}$ and KIHWAN KWON ${ }^{1,2}$ \\ ${ }^{1}$ Medical Research Institute, ${ }^{2}$ Department of Internal Medicine, Cardiology Division, School of Medicine, \\ Ewha Womans University, Yangcheon-gu, Seoul 158-710; ${ }^{3}$ Division of Cardiology, Severance Cardiovascular Hospital, \\ Yonsei University College of Medicine, Seodaemun-gu, Seoul 120-752, Republic of Korea
}

Received April 2, 2013; Accepted May 15, 2013

DOI: $10.3892 /$ ijmm.2013.1393

\begin{abstract}
Soluble receptor for advanced glycation end products RAGE (sRAGE), a secretory form of RAGE, plays an important role in suppressing RAGE signals that induce pro-inflammatory gene activation in a range of inflammatory diseases, such as Alzheimer's disease, complications of diabetes mellitus and atherosclerosis. Recent studies have suggested that fluid shear stress generated by laminar blood flow protects blood vessels from atherosclerosis, whereas low and oscillatory shear stress (OSS) generated by disturbed blood flow causes atherosclerosis. Although RAGE levels are increased in atherosclerotic plaque, the regulatory mechanisms of sRAGE in the occurrence of atherosclerotic plaque induced by disturbed blood flow remain largely unknown. This study aimed to determine the effects of sRAGE as a competitive inhibitor of RAGE in atherogenesis induced by disturbed blood flow. To determine the role of sRAGE in atherosclerosis induced by disturbed blood flow, we used a mouse model of partial carotid artery ligation using $\mathrm{ApoE}^{-/-}$and $\mathrm{C} 57 \mathrm{BL} / 6$ mice. Our results revealed that the expression of RAGE was significantly increased in the region of atherosclerotic plaque and that treatment with sRAGE attenuated the development of plaque formation. We found that the expression levels of RAGE and high mobility group box 1 (HMGB1), the agonistic ligand of RAGE, were significantly increased in human umbilical vein endothelial cells (HUVECs) under shear stress conditions induced by
\end{abstract}

Correspondence to: Professor Kihwan Kwon, Department of Internal Medicine, Cardiology Division, School of Medicine, Ewha Womans University, 911-1 Mok-dong, Yangcheon-gu, Seoul 158-710, Republic of Korea

E-mail:kankadin@ewha.ac.kr

${ }^{*}$ Contributed equally

Key words: receptor of advanced glycation end products, soluble receptor of advanced glycation end products, fluid shear stress, oscillatory shear stress, endothelial cells, atherosclerosis disturbed blood flow and suppressed following treatment with sRAGE. We further observed that treatment with sRAGE decreased the expression of vascular cell adhesion molecule-1 (VCAM-1) and markedly attenuated monocyte-endothelial cell adhesion. Taken together, our results reveal that sRAGE exerts anti-atherogenic effects by blocking the activation of the RAGE signaling pathway induced by disturbed blood flow and may thus be a potential therapeutic target for the prevention of atherosclerosis.

\section{Introduction}

Atherosclerosis is considered to be a chronic inflammatory disease (1). It has recently been suggested that atherosclerosis primarily proceeds from disturbed blood flow regions, such as various branching points and curvatures of the arteries (2). A previous study demonstrated that blood flow with laminar shear stress (LSS) increases the expression of specific genes and proteins and protects endothelial cells (ECs) against atherosclerosis, whereas shear stress generated by disturbed blood flow generally promotes atherogenesis. These data suggest that shear stress generated by disturbed blood flow is a main cause of atherosclerosis (3).

However, the exact regulatory mechanisms through which fluid shear stress affects atherogenesis remain unclear. Recently, Nam et al developed a mouse model of disturbed blood flow by partial carotid artery ligation and showed that low shear stress and oscillatory shear stress (OSS) cause endothelial dysfunction and accelerate atherosclerosis. They suggested that disturbed blood flow directly induces the development of atherosclerosis (4).

Advanced glycation end products (AGEs) and their cell surface receptors have been implicated in the pathogenesis of diabetic complications. The receptor for advanced glycation end products (RAGE) is a transmembrane receptor for AGEs and other ligands, such as high mobility group box 1 (HMGB1). The binding of RAGE and these ligands activate ECs and monocytes, leading to inflammation (5). A number of studies have demonstrated that the interaction between RAGE and its ligands leads to the development and progression of atherosclerotic disease. In animal studies, a correlation 
between RAGE and atherosclerosis has been demonstrated (6). Apolipoprotein E (apoE) and RAGE (apoE ${ }^{-/} / \mathrm{RAGE}^{-/}$) double knockout mice have been shown to develop a significantly lower number of atherosclerotic lesions compared with apoE $\mathrm{E}^{-/}$ mice with an intact expression of RAGE (7). Although RAGE expression is increased in atherosclerotic plaque, it is unknown whether arterial hemodynamics regulate RAGE expression and activation (5).

Soluble RAGE (sRAGE) is the soluble receptor form of RAGE lacking the intracellular domain through the proteolytic cleavage of RAGE or alternative RNA splicing in humans (8). Typically, sRAGE functions as a competitive inhibitor of RAGE by interacting with AGEs and other ligands, such as HMGB1, resulting in the inhibition of RAGE-induced cellular signaling, tissue damage and dysfunction (9). The administration of sRAGE to diabetic ApoE ${ }^{-/-}$mice has been shown to repress atherogenesis, as well as to stabilize established atherosclerosis $(6,10,11)$. In a model of carotid arterial injury, the interruption of RAGE-ligand interaction by treatment with sRAGE has been shown to result in the reduced proliferation of smooth muscle cells and neointimal formation in both diabetic and non-diabetic rats (12).

The regulatory mechanisms of sRAGE in the occurrence of atherosclerotic plaque induced by disturbed blood flow remain largely unknown. In this study, we demonstrate that RAGE expression is directly modulated by fluid shear stress and that sRAGE inhibits RAGE-induced pro-inflammatory responses induced by OSS. Therefore, sRAGE, as a competent inhibitor of RAGE plays a protective role during the development of atherosclerosis originating from disturbed blood flow.

\section{Materials and methods}

Cell culture and blood flow experiments. Human umbilical vein endothelial cells (HUVECs; Gibco, Paisley, Scotland, UK) were grown in Medium 200 with 5\% fetal bovine serum and low-serum growth supplement (LSGS; Cascade Biologics Inc., Portland, OR, USA) (13). U937 cells were purchased from the Korean Cell Line Bank (KCLB). Cells were cultured in RPMI-1640 (Gibco, Scotland, UK) with $10 \%$ fetal bovine serum (Gibco) and 1\% penicillin-streptomycin (Corning Cellgro, Manassas, VA, USA) at $37^{\circ} \mathrm{C}$ and $5 \% \mathrm{CO}_{2}$.

Confluent HUVECs cultured in $60-\mathrm{mm}$ dishes were exposed to fluid shear stress. Cells were exposed to flow in a cone and plate viscometer. We exposed a unidirectional steady flow (shear stress of $15 \mathrm{dyne} / \mathrm{cm}^{2}$ ) for LSS and a bidirectional disturbed flow (shear stress of $\pm 5 \mathrm{dyne} / \mathrm{cm}^{2}$ ) for OSS as previously described (14).

Model of partial carotid artery ligation. This animal study was performed in accordance with the Guidelines for Animal Experiments of the Animal Experimentation Ethics Committee of Ewha Womans University, Seoul, Korea. To investigate the function of sRAGE in blood vessels under disturbed flow conditions, we generated a mouse model of disturbed flow by partial ligation of the carotid artery as previously described (4). Male mice were ligated at 6 weeks of age. ApoE $^{-/}$and C57BL/6 mice were purchased from the Central Animal Laboratory, Inc., Seoul, Korea. Partial carotid artery ligated mice are viable and show acutely induced disturbed blood flow with low shear stress and OSS, resulting in endothelial dysfunction and atherosclerosis. All mice were fed a chow diet and provided with water ad libitum until partial ligation. Partial ligation of the left carotid artery (LCA) was carried out as described in a previous study (4).

$\mathrm{ApoE}^{-/-}$mice were treated with sRAGE $(80 \mu \mathrm{g} / \mathrm{kg}$; A\&RT Co., Korea) or PBS through intraperitoneal injection daily for 2 weeks and subsequently sacrificed. C57BL/6 mice were sacrificed at 1,3 and 7 days following partial carotid artery ligation.

Immunofluorescence staining. The mice were sacrificed and perfused with saline containing heparin $(10 \mathrm{U} / \mathrm{ml})$ through the left ventricle. The LCA and right carotid artery (RCA) were collected en bloc with the trachea and esophagus. For the cryosections, tissue was embedded in Tissue-Tek optimum cutting temperature (OCT) medium, frozen on dry ice and stored at $-80^{\circ} \mathrm{C}$ until use (15). Cross cryosections $(4 \mu \mathrm{m})$ were air-dried for $1 \mathrm{~h}$ at room temperature. The tissue sections were then placed in $10 \%$ formalin for $10 \mathrm{~min}$ at room temperature for fixation. They sections were then washed in PBS for $5 \mathrm{~min}$ to remove the OCT compound and then blocked with $10 \%$ normal donkey serum (Santa Cruz Biotechnology, Inc., Santa Cruz, CA, USA) in PBS for $2 \mathrm{~h}$. The tissue sections were then stained with rabbit anti-RAGE (1:50; Abcam, Cambridge, MA, USA) or rabbit anti-CD31 (1:50; Abcam) overnight at $4^{\circ} \mathrm{C}$. The samples were then incubated for $2 \mathrm{~h}$ in the dark with anti-rabbit secondary antibody (1:200; Santa Cruz Biotechnology, Inc.). Nuclei were counterstained with DAPI (100 ng/ml; Santa Cruz Biotechnology, Inc.) for $8 \mathrm{~min}$ in the dark. The tissue sections were then routinely stained with hematoxylin and eosin (H\&E) (16). The slides were viewed on an Olympus BX51 microscope (Olympus America Inc., Melville, NY, USA).

Immunohistochemical staining. Cryosections were fixed in $10 \%$ formalin for $10 \mathrm{~min}$ and blocked with peroxide block (Cell Marque, Rocklin, CA, USA) for $10 \mathrm{~min}$ at room temperature. Samples were incubated with rabbit anti-RAGE antibodies (1:50, 1:100; Abcam) or rabbit anti-CD31 (1:50; Abcam) for $1 \mathrm{~h}$ at room temperature. To visualize primary antibodies, the Polink-2 Plus HRP Detection kit (Golden Bridge International, Inc., Mukilteo, WA, USA) was used. Nuclei were counterstained with Mayer's hematoxylin (Merck KGaA, Darmstadt, Germany) (6). Oil red O staining was carried out with using frozen sections as previously described (17).

Monocyte adhesion assay. HUVECs were treated with sRAGE $(1 \mu \mathrm{g} / \mathrm{ml})$ followed by exposure to static, laminar $\left(15 \mathrm{dyne} / \mathrm{cm}^{2}\right)$ and oscillatory flow $\left( \pm 5 \mathrm{dyne} / \mathrm{cm}^{2}\right)$ for $24 \mathrm{~h}$. For the positive controls, cells were treated with $10 \mathrm{ng} / \mathrm{ml}$ tumor necrosis factor- $\alpha$ (TNF- $\alpha, 10 \mathrm{ng} / \mathrm{ml}$ ) for $6 \mathrm{~h}$. The medium was then removed, and U937 cells were added to the dishes and incubated for $30 \mathrm{~min}$ at $37^{\circ} \mathrm{C}$. The unbound cells in the dishes were then removed by washing 3 times with serum-free medium. The adherent cells were counted in 5 randomly selected optical fields in each well. Phase-contrast microphotographs of the cells in the plates were taken using an Olympus CKX41 inverted microscope (Olympus America Inc.).

RNA isolation, reverse-transcription polymerase chain reaction $(P C R)$. Total RNA was isolated from the cultured 
A

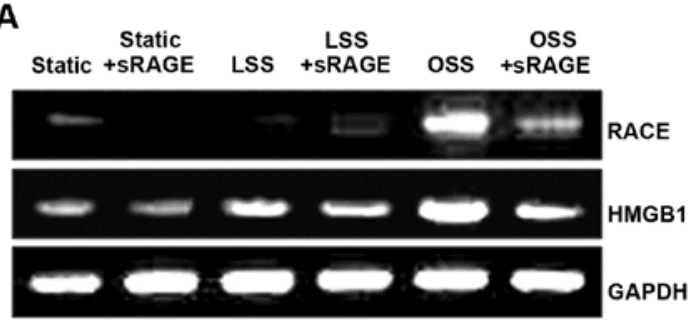

C

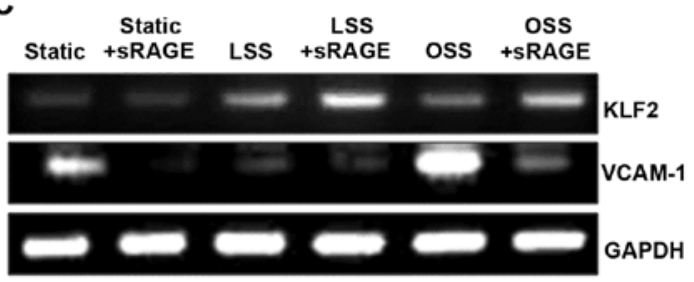

B


Figure 1. Soluble receptor for advanced glycation end products (sRAGE) regulates flow-mediated Krüppel-like factor 2 (KFL2) and VCAM-1 expression through the RAGE-dependent pathway. (A-D) Human umbilical vein endothelial cells (HUVECs) were exposed for $6 \mathrm{~h}$ to flow or to static conditions following pre-treatment with sRAGE $(1 \mu \mathrm{g} / \mathrm{ml})$. mRNA was extracted from the cell lysates and real-time reverse-transcription plymerase chain reaction (RT-PCR) with the primers of RAGE, high mobility group box 1 (HMGB1), KLF2, VCAM-1 and GAPDH (internal control) was carried out as described in Materials and methods. Representative images and quantitative data are shown $(\mathrm{n}=4)$.

HUVECs by the use of a Total RNA Isolation kit (Qiagen Inc., Hilden, Germany) (13). First-strand cDNA was synthesized using the Super Script III first-strand synthesis system (Invitrogen, Carlsbad, CA, USA). cDNA was amplified by PCR for 30 cycles (Eppendorf AG, Hamburg, Germany). The following oligonucleotide primers were used in this study: human RAGE sense, 5'-AGCGGCTGGAATGGAAACTG AACA-3' and antisense, 5'-GAAGGGGCAAGGGCACA CCATC-3'; human HMGB1 sense, 5'-GCGACTCTGTGCC TCGCTGA-3' and antisense, 5'-ACGGGCCTTGTCC GCTTTTGC-3'; human VCAM-1 sense, 5'-GGCCTCAGTC AGTGTGA-3' and antisense, 5'-AACCCCATTCAGCG TCA-3'; human GAPDH sense, 5'-GAGTCAACGGATTT GGTCGT-3' and antisense, 5'-TTGATTTTGGAGGG ATCTCG-3'. The experiments were repeated 3 times.

Statistical analysis. All data are expressed as the means \pm SEM from least 3 independent experiments with different sample sizes. A paired t-test was used to assess the significance of the results between 2 groups. Differences between 3 or more groups were analyzed by contrast analysis, using Super ANOVA. A p-value $<0.05$ was considered to indicate a statistically significant difference.

\section{Results}

SRAGE represses RAGE-dependent inflammatory effects under OSS conditions. To determine whether sRAGE is involved in the RAGE-dependent pathway in ECs, we treated HUVECs with sRAGE ( $1 \mu \mathrm{g} / \mathrm{ml}$ of EC medium) and subsequently exposed the cells to LSS (fluid shear stress, $15 \mathrm{dyne} / \mathrm{cm}^{2}$ ) or OSS (shea stress, $\pm 5 \mathrm{dyne} / \mathrm{cm}^{2}$ ) for $24 \mathrm{~h}$.
The cell lysates were collected, and the mRNA expression of RAGE was analyzed by RT-PCR. As shown in Fig. 1A, sRAGE significantly inhibited the OSS-induced expression of RAGE in the HUVECs. We also observed that sRAGE reduced the mRNA expression level of HMGB1, a ligand of RAGE. These results suggest that sRAGE plays an important role in the RAGE-dependent pathway as a competent inhibitor of RAGE under OSS conditions.

LSS plays a critical role in enhancing the survival of ECs and regulating vascular tone; it also exerts anti-inflammatory and anti-atheroscleroic effects (18-22). Previous studies have shown that OSS generally upregulates genes with proinflammatory and atherogenic properties (22-25). These effects occur through the LSS-dependent induction of certain genes, such as Krüppel-like factor -2 (KLF2) (26-29). KLF-2 is induced by LSS, which in turn regulates a number of flowresponsive genes $(26,27,30)$. Furthermore, KLF2 has been shown to regulate leukocyte adhesion to the endothelium by downregulating the expression of adhesion molecules that recruit leukocytes, such as vascular cell adhesion molecule-1 (VCAM-1) (26,31,32). We used RT-PCR to analyze the expression of KLF2 and VCAM-1. The mRNA expression level of KLF2 was increased by LSS; however, it was significantly reduced by OSS. However, sRAGE significantly upregulated KLF2 expression in HUVECs under OSS (Fig. 1B). By contrast, when the cells were treated with sRAGE, the OSS-induced VCAM-1 expression was markedly attenuated (Fig. 1B). Takeb together, these data demonstrate that sRAGE inhibits RAGE-dependent inflammation induced by OSS.

sRAGE suppresses OSS-induced inflammation in ECs. KLF2 exerts anti-inflammatory effects in ECs by inhibiting the 



B

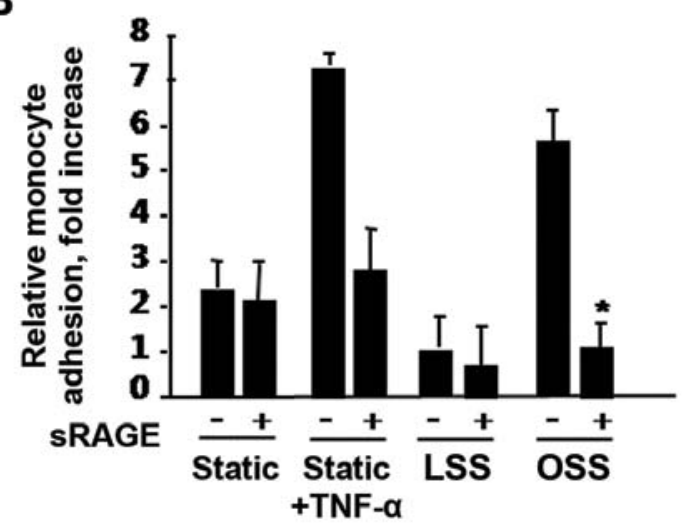

Figure 2. Soluble receptor for advanced glycation end products (sRAGE) suppresses oscillatory shear stress (OSS)-induced monocyte adhesion to endothelial cells. (A and B) Treatment with sRAGE attenuated OSS-induced inflammation. Human umbilical vein endothelial cells (HUVECs) were subjected to flow or to static conditions for $24 \mathrm{~h}$ following pre-treatment with sRAGE $(1 \mu \mathrm{g} / \mathrm{ml})$ followed by treatment with TNF- $\alpha$ or the vehicle for $6 \mathrm{~h}$. U937 monocytes were added to the HUVECs and incubated for $30 \mathrm{~min}$. HUVECs were washed 3 times. The adherent monocytes were counted in 5 randomly optical fields in each dish. "P<0.05, OSS vs. OSS + sRAGE. Error bars represent the means \pm SEM.

expression of VCAM-1, which regulates the recruitment of monocytes to the ECs (26). To determine the potential role of sRAGE in repressing the OSS-induced pro-inflammatory effects, we examined monocyte adhesion to HUVECs under static, LSS and OSS conditions with or without treatment with sRAGE (Fig. 2). ECs challenged with the pro-inflammatory cytokine, TNF- $\alpha$, were used as the positive controls. As shown in Fig. 2, the TNF- $\alpha$ and OSS-stimulated cells exhibited augmented monocyte adhesion. By contrast, the sRAGE-treated ECs displayed significantly less monocyte adhesion even under static conditions with TNF- $\alpha$ treatment and OSS. Taken together, these results indicate that sRAGE regulates monocyte-EC adhesion, stimulated by OSS.

Partial carotid artery ligated mice have enhanced RAGE expression in regions of disturbed blood flow. To directly examine variations in RAGE expression under OSS conditions in vivo, we used a mouse model of disturbed blood flow with low OSS induced by partial carotid artery ligation (4).

As shown in Fig. 3, RAGE expression was markedly increased in the partially ligated LCA, whereas there was a significantly lower expression level of RAGE in the nonligated RCA (Fig. 3A). To confirm whether shear stress modulates the expression of RAGE in ECs, we performed immunohistochemical staining on tissues from partially ligated C57BL/6 mice (Fig. 3B). The endothelial layer was stained with CD31 as an EC marker. The levels of RAGE expression were significantly enhanced in the partially ligated LCA compared with the non-ligated RCA. This suggests that shear stress modulates the expression of RAGE in the vasculature. These findings are consistent with those from previous studies, showing that RAGE transcript levels are highly expressed at the site of disturbed flow compared to the site of normal blood flow in the arteries of swine (33). In our study, the expression of RAGE was increased in the LCA even as early as 1 day following ligation (Fig. 3C). On day 7, RAGE expression was markedly upregulated in the LCA compared with the RCA.

SRAGE reduces atherosclerotic plaque formation in vivo. To explore the role of SRAGE in disturbed flow-induced atherosclerotic plaque formation in vivo, we used a mouse model of partial carotid artery ligation using $\mathrm{ApoE}^{-/-}$mice. The mice were fed a high-fat diet for 2 weeks following surgery. sRAGE $(80 \mu \mathrm{g} / \mathrm{kg})$ or PBS was injected into the intraperitoneal space, daily for 2 weeks. As shown in Fig. 4, the LCA of the control group showed severe atherosclerotic plaque formation as determined by Oil red O staining (Fig. 4A). However, the LCA of the sRAGE-treated group showed significantly reduced plaque formation (Fig. 4B). To better understand the effect of sRAGE 
A
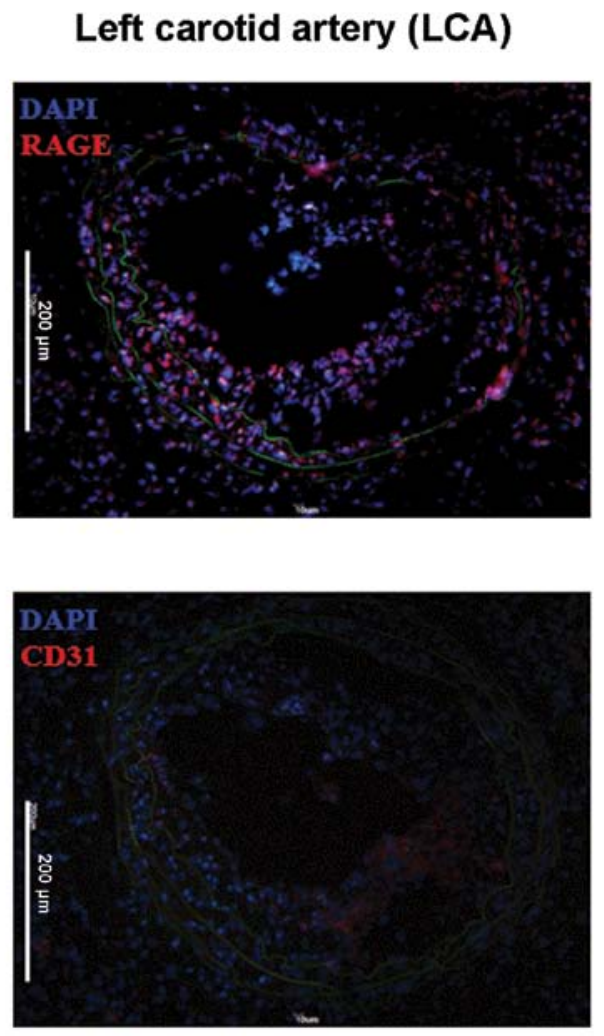

B
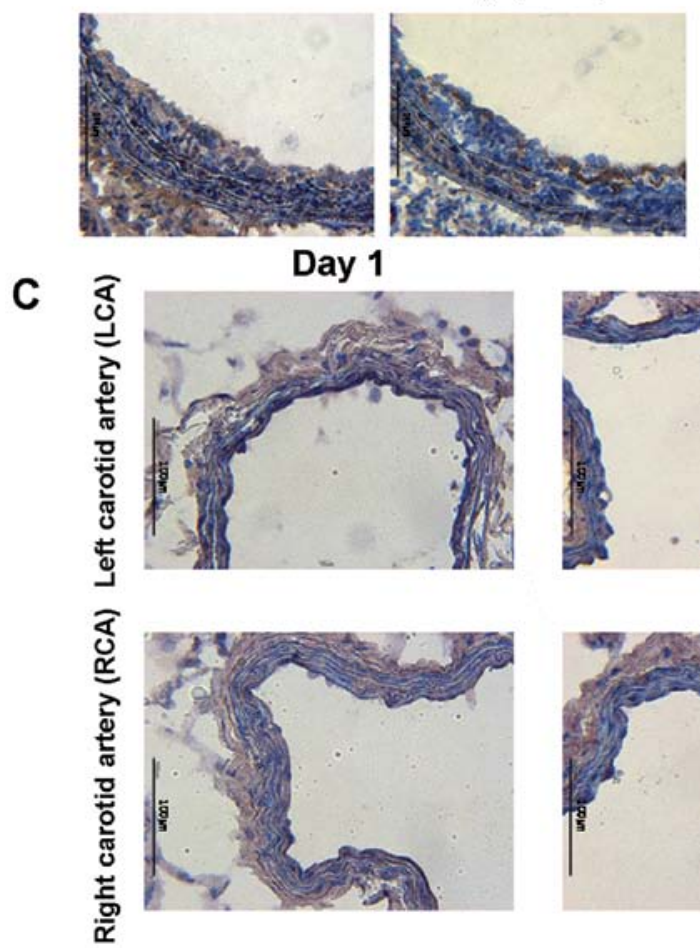

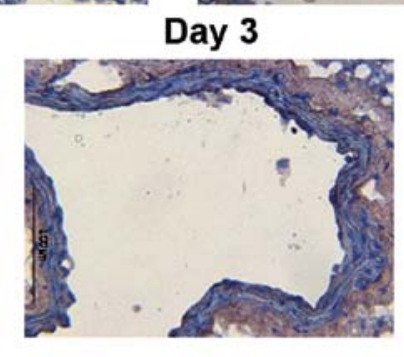

\section{Right carotid artery (RCA)}
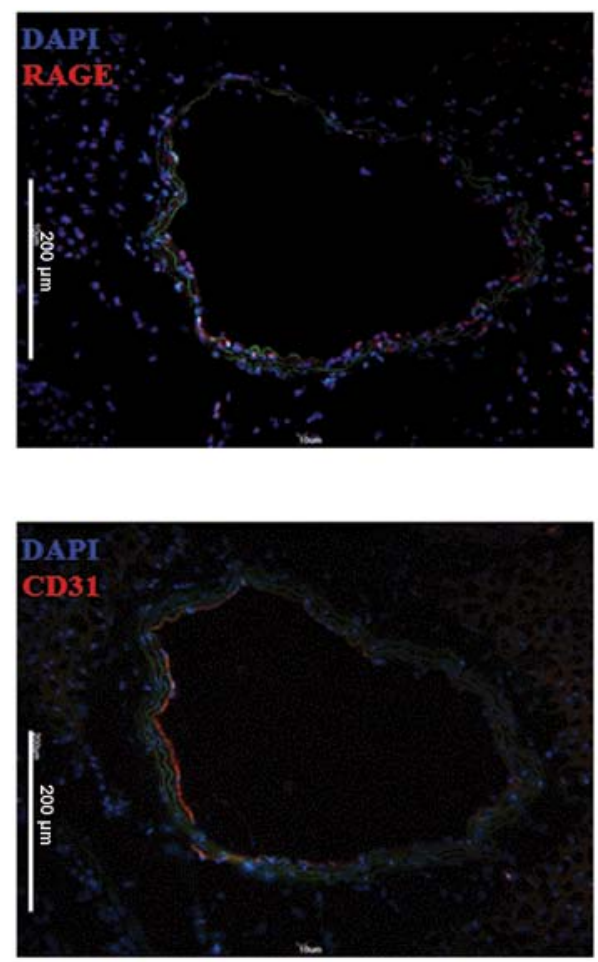

\section{Right carotid artery (RCA)}
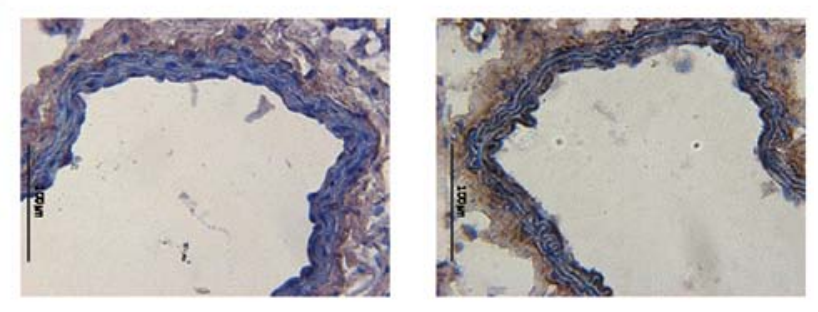

Figure 3. Partial carotid artery ligated mice have an enhanced expression of receptor for advanced glycation end products (RAGE) in the disturbed flow region. (A) Immunofluorescence staining for RAGE and CD31 on tissues from the left carotid artery (LCA) and right carotid artery (RCA) at 14 days in the ApoE ${ }^{-1}$ mice. Sites of RAGE expression in endothelial cells were confirmed by the co-localization of RAGE expression with CD31. Red color indicates RAGE- or CD31-positive staining; blue indicates 4',6-diamidino-2-phenylindole (DAPI) nuclear staining. Magnification, x20. (B and C) The carotid arteries of C57BL/6 mice were subjected to immunohistochemical staining to detect RAGE antigen. (B) Frozen sections from LCA and RCA on day 14 were stained for RAGE. (C) The induction of RAGE expression in the carotid arteries was also examined on days 1,3 and 7. Original magnification, x20.

in disturbed flow-induced atherosclerotic plaque formation, the severity of stenosis (Fig. 4C) and plaque area (Fig. 4D) were compared between the control and sRAGE-treated group. The non-ligated RCA in both groups did not show any evidence of luminal stenosis and atherosclerotic plaque formation. In contrast to the RCA, the ligated LCA of the control group 



Figure 4. Soluble receptor for advanced glycation end products (sRAGE) reduces atherosclerotic plaque formation in vivo. ApoE ${ }^{-/}$mice were partially ligated and fed a high-fat diet for 2 weeks. The mice were then treated with (A) PBS or (B) sRAGE (80 $\mu \mathrm{g} / \mathrm{kg}$ ) for 2 weeks. (A and B) Shown are representative images from at least 6 experiments. Frozen sections from the left carotid artery (LCA) and right carotid artery (RCA) were stained with hematoxylin and eosin (H\&E). (C and D) To better understand the effect of sRAGE in disturbed flow-induced atherosclerotic plaque formation, (C) the severity of stenosis and (D) the plaque area were compared between the control and sRAGE-treated group

showed severe stenosis and a significantly increased plaque area, while the sRAGE-treated group showed significantly reduced luminal stenosis and plaque area. These results suggest that sRAGE inhibits the development of atherosclerotic plaque induced by disturbed blood flow.

\section{Discussion}

The novel finding of the present study is that sRAGE plays a critical role as an inhibitor of atheromatous plaque formation induced by disturbed blood flow. First, sR AGE repressed the pro-inflammatory response induced by OSS through the downregulation of RAGE, HMGB1 and VCAM-1 expression. According to a previous study, HMGB1, the specific ligand for RAGE, is upregulated at sites of activated ECs during vascular inflammation (9). In the current study, the expression of HMGB1 under OSS conditions was significantly inhibited following treatment with sRAGE. Monocyte binding was also inhibited following treatment with sRAGE through the decreased expression of VCAM-1. RAGE expression itself was decreased by sRAGE under OSS conditions. Therefore, these findings suggest that sRAGE may be an important inhibitor of vascular inflammatory responses generated by disturbed blood flow.

Furthermore, the expression of KLF2, an anti-inflammatory factor, was induced following treatment with sRAGE under OSS conditions. This result indicates that sRAGE induces the expression of atheroprotective factors which protect cells in a pro-inflammatory environment, such as during the early atherosclerotic process.

Second, we demonstrated that sRAGE suppresses OSS-induced inflammatory responses, such as monocyte-EC adhesion in in vitro experiments, as well as the development of atherosclerotic plaque in partially ligated carotid arteries of $\mathrm{ApoE}^{-/-}$mice in vivo. We showed that RAGE expression was enhanced in the LCA, particularly in the endothelium of the lesion of disturbed blood flow, and that the administration of sRAGE suppressed atheromatous plaque formation in the partially ligated carotid arteries of $\mathrm{ApoE}^{-/-}$mice. These findings are consistent with those from previous studies, showing that the treatment of $\mathrm{ApoE}^{-/-}$mice with sRAGE reduced the development and progression of atherosclerosis in a dose-dependent manner and that the progression of atherosclerosis was halted by the administration of sRAGE in diabetic $\mathrm{ApoE}^{-/-}$mice $(5,6)$. However, these previous studies did not determine the correlation between disturbed blood flow, RAGE expression, sRAGE and atheromatous plaque formation. By comparison, in our study, RAGE expression was increased by disturbed blood flow and it was suppressed following treatment with sRAGE. Furthermore, we showed that sRAGE suppressed atheromatous plaque formation induced by disturbed blood flow. Blood vessels, particularly ECs are consistently exposed to fluid shear stress, the dragging force generated by blood flow. Certain studies have suggested 
that fluid shear stress modulates vascular homeostasis and the focal distribution of atherosclerosis by regulating endothelial gene expression $(19,34)$. Investigating the regulation of blood flow-mediated genes is therefore mandatory in order to gain a better understanding of atheroprotection.

To our knowledge, this is the first study demonstrating that sRAGE regulates the process of atherogenesis induced by disturbed blood flow. Previous studies using static-cultured ECs have demonstrated that inflammatory signaling is downregulated by sRAGE through the blockade of RAGE $(35,36)$. However, in this study, we demonstrate that fluid shear stress itself regulates the expression of RAGE and its ligand, HMGB1. Another major finding of this study was that sRAGE plays a crucial role in the regulation of the RAGE-dependent pathway involving the expression of pro-inflammatory and anti-inflammatory genes (VCAM-1 and KLF2) even in the absence of additional RAGE ligands, such as HMGB1 and certain S100 family members. This study unveils a novel role of sRAGE in regulating atherogenesis in response to hemodynamic forces, and thus provides the foundation for novel approaches for the treatment of atherosclerosis.

\section{Acknowledgements}

The present study was supported by the Bio and Medical Technology Development Program of the National Research Foundation (NRF) funded by the Korean government (MEST) (no. 2011-0019695).

\section{References}

1. Ross R: Atherosclerosis - an inflammatory disease. N Engl J Med 340: 115-126, 1999

2. Chiu JJ, Usami S and Chien S: Vascular endothelial responses to altered shear stress: pathologic implications for atherosclerosis. Ann Med 41: 19-28, 2009.

3. Chiu JJ and Chien S: Effects of disturbed flow on vascular endothelium: pathophysiological basis and clinical perspectives. Physiol Rev 91: 327-387, 2011.

4. Nam D, Ni CW, Rezvan A, Suo J, Budzyn K, Lianos A, Harrison D, Giddens D and Jo H: Partial carotid ligation is a model of acutely induced disturbed flow, leading to rapid endothelial dysfunction and atherosclerosis. Am J Physiol Heart Circ Physiol 297: H1535-H1543, 2009.

5. DeVerse JS, Bailey KA, Jackson KN and Passerini AG: Shear stress modulates RAGE-mediated inflammation in a model of diabetes-induced metabolic stress. Am J Physiol Heart Circ Physiol 302: H2498-H2508, 2012.

6. Lindsey JB, Cipollone F, Abdullah SM and McGuire DK: Receptor for advanced glycation end-products (RAGE) and soluble RAGE (sRAGE): cardiovascular implications. Diab Vasc Dis Res 6: 7-14, 2009.

7. Harja E, Bu DX, Hudson BI, Chang JS, Shen X, Hallam K, Kalea AZ, Lu Y, Rosario RH, Oruganti S, Nikolla Z, Belov D, Lalla E, Ramasamy R, Yan SF and Schmidt AM: Vascular and inflammatory stresses mediate atherosclerosis via RAGE and its ligands in apoE ${ }^{-/-}$mice. J Clin Invest 118: 183-194, 2008.

8. Schlueter C, Hauke S, Flohr AM, Rogalla P and Bullerdiek J: Tissue-specific expression patterns of the RAGE receptor and its soluble forms - a result of regulated alternative splicing? Biochim Biophys Acta 1630: 1-6, 2003.

9. Herold K, Moser B, Chen Y, Zeng S, Yan SF, Ramasamy R, Emond J, Clynes R and Schmidt AM: Receptor for advanced glycation end products (RAGE) in a dash to the rescue: inflammatory signals gone awry in the primal response to stress. J Leukoc Biol 82: 204-212, 2007.

10. Park L, Raman KG, Lee KJ, Lu Y, Ferran LJ Jr, Chow WS, Stern D and Schmidt AM: Suppression of accelerated diabetic atherosclerosis by the soluble receptor for advanced glycation endproducts. Nat Med 4: 1025-1031, 1998.
11. Bucciarelli LG, Wendt T, Qu W, Lu Y, Lalla E, Rong LL, Goova MT, Moser B, Kislinger T, Lee DC, Kashyap Y, Stern DM and Schmidt AM: RAGE blockade stabilizes established atherosclerosis in diabetic apolipoprotein E-null mice. Circulation 106: 2827-2835, 2002.

12. Zhou Z, Wang K, Penn MS, Marso SP,Lauer MA, Forudi F, Zhou X, Qu W, Lu Y, Stern DM, Schmidt AM, Lincoff AM and Topol EJ: Receptor for AGE (RAGE) mediates neointimal formation in response to arterial injury. Circulation 107: 2238-2243, 2003.

13. Ha CH, Wang W, Jhun BS, Wong C, Hausser A, Pfizenmaier K, McKinsey TA, Olson EN and Jin ZG: Protein kinase D-dependent phosphorylation and nuclear export of histone deacetylase 5 mediates vascular endothelial growth factor-induced gene expression and angiogenesis. J Biol Chem 283: 14590-14599, 2008.

14. Jin ZG, Ueba H, Tanimoto T, Lungu AO, Frame MD and Berk BC: Ligand-independent activation of vascular endothelial growth factor receptor 2 by fluid shear stress regulates activation of endothelial nitric oxide synthase. Circ Res 93: 354-363, 2003.

15. Dave SH, Tilstra JS, Matsuoka K, Li F, DeMarco RA, BeerStolz D, Sepulveda AR, Fink MP, Lotze MT and Plevy SE: Ethyl pyruvate decreases HMGB1 release and ameliorates murine colitis. J Leukoc Biol 86: 633-643, 2009.

16. Sasaki T, Kuzuya M, Cheng XW, Nakamura K, Tamaya-Mori N, Maeda K, Kanda S, Koike T, Sato K and Iguchi A: A novel model of occlusive thrombus formation in mice. Lab Invest 84: 1526-1532, 2004

17. Zhou J, Lhotak S, Hilditch BA and Austin RC: Activation of the unfolded protein response occurs at all stages of atherosclerotic lesion development in apolipoprotein E-deficient mice. Circulation 111: 1814-1821, 2005.

18. Berk BC: Atheroprotective signaling mechanisms activated by steady laminar flow in endothelial cells. Circulation 117: 1082-1089, 2008.

19. Davies PF: Flow-mediated endothelial mechanotransduction. Physiol Rev 75: 519-560, 1995.

20. Gimbrone MA Jr, Topper JN, Nagel T, Anderson KR and Garcia-Cardena G: Endothelial dysfunction, hemodynamic forces, and atherogenesis. Ann NY Acad Sci 902: 230-240, 2000.

21. Boo YC and Jo H: Flow-dependent regulation of endothelial nitric oxide synthase: role of protein kinases. Am J Physiol Cell Physiol 285: C499-C508, 2003.

22. Chien S: Mechanotransduction and endothelial cell homeostasis: the wisdom of the cell. Am J Physiol Heart Circ Physiol 292: H1209-H1224, 2007.

23. Shyy YJ, Hsieh HJ, Usami S and Chien S: Fluid shear stress induces a biphasic response of human monocyte chemotactic protein 1 gene expression in vascular endothelium. Proc Natl Acad Sci USA 91: 4678-4682, 1994.

24. Kraiss LW, Geary RL, Mattsson EJ, Vergel S, Au YP and Clowes AW: Acute reductions in blood flow and shear stress induce platelet-derived growth factor-A expression in baboon prosthetic grafts. Circ Res 79: 45-53, 1996.

25. Wilcox JN, Smith KM, Williams LT, Schwartz SM and Gordon D: Platelet-derived growth factor mRNA detection in human atherosclerotic plaques by in situ hybridization. J Clin Invest 82: 1134-1143, 1988.

26. SenBanerjee S, Lin Z, Atkins GB, Greif DM, Rao RM, Kumar A, Feinberg MW, Chen Z, Simon DI, Luscinskas FW, Michel TM, Gimbrone MA Jr, Garcia-Cardena G and Jain MK: KLF2 is a novel transcriptional regulator of endothelial proinflammatory activation. J Exp Med 199: 1305-1315, 2004.

27. Parmar KM, Larman HB, Dai G, Zhang Y, Wang ET, Moorthy SN, Kratz JR, Lin Z, Jain MK, Gimbrone MA Jr, and Garcia-Cardena G: Integration of flow-dependent endothelial phenotypes by Kruppel-like factor 2. J Clin Invest 116: 49-58, 2006.

28. Lee JS, Yu Q, Shin JT, Sebzda E, Bertozzi C, Chen M, Mericko P, Stadtfeld M, Zhou D, Cheng L, Graf T, MacRae CA, Lepore JJ, Lo CW and Kahn ML: Klf2 is an essential regulator of vascular hemodynamic forces in vivo. Dev Cell 11: 845-857, 2006.

29. Atkins GB and Jain MK: Role of Kruppel-like transcription factors in endothelial biology. Circ Res 100: 1686-1695, 2007.

30. Dekker RJ, van Soest S, Fontijn RD, Salamanca S, de Groot PG, VanBavel E, Pannekoek H and Horrevoets AJ: Prolonged fluid shear stress induces a distinct set of endothelial cell genes, most specifically lung Kruppel-like factor (KLF2). Blood 100: 1689-1698, 2002 
31. Tsao PS, Buitrago R, Chan JR and Cooke JP: Fluid flow inhibits endothelial adhesiveness. Nitric oxide and transcriptional regulation of VCAM-1. Circulation 94: 1682-1689, 1996.

32. Fledderus JO, van Thienen JV, Boon RA, Dekker RJ, Rohlena J, Volger OL, Bijnens AP, Daemen MJ, Kuiper J, van Berkel TJ, Pannekoek $\mathrm{H}$ and Horrevoets AJ: Prolonged shear stress and KLF2 suppress constitutive proinflammatory transcription through inhibition of ATF2. Blood 109: 4249-4257, 2007.

33. Passerini AG, Polacek DC, Shi C, Francesco NM, Manduchi E, Grant GR, Pritchard WF, Powell S, Chang GY, Stoeckert CJ Jr and Davies PF: Coexisting proinflammatory and antioxidative endothelial transcription profiles in a disturbed flow region of the adult porcine aorta. Proc Natl Acad Sci USA 101: 2482-2487, 2004.

34. Berk BC, Abe JI, Min W, Surapisitchat J and Yan C: Endothelial atheroprotective and anti-inflammatory mechanisms. Ann NY Acad Sci 947: 93-111, 2001.
35. Mullins GE, Sunden-Cullberg J, Johansson AS, Rouhiainen A, Erlandsson-Harris H, Yang H, Tracey KJ, Rauvala H, Palmblad J, Andersson $\mathbf{J}$ and Treutiger $\mathrm{CJ}$ : Activation of human umbilical vein endothelial cells leads to relocation and release of highmobility group box chromosomal protein 1 . Scand J Immunol 60: 566-573, 2004

36. Frommhold D, Kamphues A, Hepper I, Pruenster M, Lukic IK, Socher I, Zablotskaya V, Buschmann K, Lange-Sperandio B, Schymeinsky J, Ryschich E, Poeschl J, Kupatt C, Nawroth PP, Moser M, Walzog B, Bierhaus A and Sperandio M: RAGE and ICAM-1 cooperate in mediating leukocyte recruitment during acute inflammation in vivo. Blood 116: 841-849, 2010. 